Per ispiegare tali fennmeni occorre tener conto delle volocita di propagazione di certi ragri diversamente polariz. zati, forse anzi di ma doppia rifrazone elitica. Na su questa questione non aggiungo per ora nessun' alta indicazione.

\title{
EIVISTA
}

Comptes Kendus. T. CXXIX, Settembre a Dicembre 1899.

Nikoladive (De) W. Su diverso esperienze lestinate a confermu's l'ipotesi di Ampere, relativa alla direzione dell' caione elementare elettromagnetica (pp. 475.477). .- Cno dei due sistemi, su cui l'A. ha esperimentato ó formato da un'elettrocalamita tabulare che puó ruotare attorno a un filo di sospensione, e del cai circuito una estremità é immersa nel marcurio di una vasehetta posta al disotto, mentre l'altu $\dot{\theta}$ in comunicazione per mezzo di un filo con una vaschetta anulare legata invariabilmente all'elettro. L'altro sistema è costituito da un tubo vuoto percorso dal basso ju alto da una corrente che paò scendere per un' asta di cttone, posta all' interno del tubo, e il tubo stesso o pinzzato fra le due ar'nature di un' elettrocalamita di Faralay. Dalle esperienze miuntamente descritte l'A. è condotto alla couclusione che $\mathrm{i}$ feno. meni si producono sempre come se $\mathrm{i}$ campi magnotici di tutte le correnti lineari di nu sistema solendidale sussistessero indipendentemente gli uni dagli altri, malgrado l'assenza di forza magnetica all' esterno del solenoide.

LIPPMANN G. Metodo per la mess.t a punto di un collimatore (pp. 569-570). - Il metodo che l'A. consigiia come preciso per mettere a punto un collimatore consiste nell' interporre fra il colJimatore e un cannocchiale ausiliare, con cai si osserva l' immagine della fenditura rischiaruta, un sistema di due lamine.di vetro a facce piane e parallele, ad angolo retto fra loro e inclinate di circa $45^{\circ}$ sul fascio laninoso. I' intersezione delle due lamine deve essere parallela alla fenditura.

Il collimatore è a punto, cioé la fenditura è nel piano focale dell' obbiettivo, quando, variando opportunamente la distaura della fenditura dall'obbiettivo, le due imagini di ossa, ottenute per rifrazione attravelso il sistema delle due lamine, sembrano confondersi. 
L'A. nota che potrebbe adoperarsi invece del sistema di due lamine, una lamina sola interposta sul passaggio della metá del fascio luminoso, a condizione che questa lamina avesse ficce piane ed esattamento parallele. Quest' ultima condizione, difficilmente realizzabile, rende questo secondo metodo ineno sicuro del primo.

LeCarme J. e L. Esperienze di telegrafia senza fili eseguite fra Chamonix e il. vertice del Monte Bianco (pp. 589-591). - Con queste esperienze gli $A$. si sono proposti di vedere:

1." se la telegrafia senza fili $\dot{o}$ praticamente possibile in montagua;

$2^{\circ}$ se l'elettricita atmosferica nuoce alle comunicazioni;

3. " se l' afficio del filo di terra persiste malgrado l'assenza di acqua allo stato liquido sul suolo.

A questo scopo gli A. hanno posto il trasmeltitore e il ricevi. tore su due alture distanti circa $12 \mathrm{~km}$; $\theta$ differenti in altezza di $3350 \mathrm{~m}$. I risultati furono i seguenti :

$1 .^{0}$ I segnali non furono ben netti che per una distanza fra le sfere dell'oscillatore di $2 \mathrm{~cm}$.

2. ${ }^{0}$ L'asseuza dell' acqua allo stato liquido non impedi lo comunicazioni.

3. ${ }^{0}$ Non jmpedirono i segnali neppure le nubi interposte fra i due luoghi.

4." I' elettricità atmosferion sebbene avesso fatto funzionare l'apparecchio a più riprese, non plodusse un'azione capace di nuocere alla telegrafia pratica.

Gli A. osservarono infine che il funzionamento della luce elettrica agisce con intensith sull' apparechio, essendo Ioro riuscito jupossibile comunicare durante l' illumiuazione di Chamonix, presso cui si trovava il trasmettitore.

Buguet A. e Crabaco V. Ampollis raliografica ad antica. todo freddo (pp. 591-593). - L' anticatodo del tubo costruito dagli A. è formato da un grosso tubo di platino saldato diretamente al vetro dell' anpolla, e circondato, fioo al centro di essa, da un manicotio di votro Alia sua estremita interna il tabo di platino $\dot{e}$ tagliato a fischio, e nua lamina di platino saldata a questa estremitá la chiude emeticamente. Questa estromita st ssa è inoltre circondata $d a$ un collaretto metallico lestinato ad arrestare i raggi catodici estranei al fascio utile. All' estremita esterna del tubo di platino si trova un piccolo serbatoio di acqua. Questo tubo può ricovere, secondo gli A., senza che l'anticatudo arrossi e senza che il vunto subisca alcuna modificazione, le scariche delle bobine più potenti, messe in azione dagli interruttori i più rapidi. 
DEBLgRNe A. Su una nuona materia radio-attiva (pp. 593595). - L'A. in base agli studi del Carie provanti che l'emissiune dei raggi constatati uella pechblende (pecurano) è dovuta oltre che all' uranio anche al polrnio e al radio, e per gli studi del Demarcay mostranti che la proprieta di emettere tali radiazioni può ben servire a calatterizare degli elementi chimici, ha ricercato se in quel minerale esistevano altri corpi radianti. Studiando principal nente $\mathrm{i}$ corpi le cui soluzjoni non precipitano per l'idrogeno solforato e precipitano completamente per l'ammoniaca, ha constatato che le parti contonenti il titano e corpi analoghi mostrauo la radio-attivita a un grado intensissisno, e ha inoltro ottenuto an'altra materia, le cui soluzioni presentano le principali proprictà analitiche del titano, e che emette raggi di nn'attività cento mila volte piu grande di quella dell' uranio. Per le radiazioni emesse questa sostanza è comparabile al polonio e al radio, dai quali peró differisce per le proprieti chimiche. Queste radiazioni rendono $i$ gas capaci di soaricare i corpi elottrizzati ; eccitano la fosforescenza del platino-cianuro di bario e impressionano le lastre fotografiche. Questa materia si distingue subito dal ratio perchè non è spontaneamente liminosa, come lo sono, nell'asuarita, j composti del radio.

Turpain A. Sulla propagazione delle oscillazioni elettriche nei mezzi dielettrici (pp. 670-672). - Dne sono le toorie sulla propagazione delle oscillazioni elettriche nei dielettrici: quella del Maxwell, per la quale $i$ fenomeni luminosi e i fenomeni elettrici souo sogretti allo stesso meccanisino, e quindi le onile si propagano per flussi esclusivanente trasversali, e quella di Helmholtz, modificata da Duhem, secondo oui le oscillazioni elettrichs si propagano per flussi trasversali e longitadinali. L'A. nelle sue esperierze avendo trovato che i] risuonatore a seconda delln posizione occopata, posizioni che ha gia indicato in un altro lavoro '), $\dot{e}$ sensibile o ai soli flussi longitudinali o ai soli flussi trasversali, conclude in completo accordo colin teoria Helmholtz-Duhem.

Branly E. Trasmissione delle onde erziane altraverso $i$ liquidi (pp. 672-675). - L'A. in questa Nota ha esposto i risultati dei suoi studi sull' assorbinento delle onde erzinne per parte di strati liquicli di $0 ", 20$ di spessoro. L'apparecchio adoperato era una cassa cubica di $60 \mathrm{~cm}$. di lato, contenente il liquido da studiarsi. Le pareti della cassa erano di vetro spesso, all' infuori di una, fortnata da una spessa placea di zinco, portinte un' apertara quadrata, attraverso cni passava una piccola cassa di legno conte-

1) C, K. :1 Gontaio 1598 . 
neute il ricevitore. L' apertara veniva quindi ermeticamente chiusa per Inezzo di viti. Il radiatore era posto di fronte alla parete di vetro opposta a quella metallica. Il ricevitore era formato da un radioconduttore intercalato nel circuito di una Leclanché $\theta$ di un relais, il cui circuito secondario comprendeva una soneria. I risultati ottenuti dall'A. mostrano che l'acqua distillata e l'acqua di sorgente esercitano un assorbimento issai superiore a quello esercitato dall' aria $\theta$ dall' olio. L'acqua del mare presenta un assorbimento completo sotto uuo spessore notevolmente inferiore a $20 \mathrm{~cm}$. Il solfato di zinco, di rame, di soda presentano assorbimenti minori, comparabili peró ancora a quello del cloruro di sodio.

La grandeza del recipiento limitarono le esperienzo, sulle quali l'A. si riserva di tornare.

Ro'HÉ E. Sull' interruttore elettrolitico di Wehnelt (fi). 675677). - Facendo variare la resistenza del circuito, e mantenendo costante la f. e. m., l'A. ha osservato vari fenomeni interessanti presentati dell' interruttore Wehnelt. Dando alla resistenza un valore grandissimo la corrente aveva una debole intensita, ma era continua e sensibilmente cjstante. L'apparecchio in questo caso non funzionava come interruttore.

Diminuendo la resistenza, l'intensiti cresceva secondo la legge di Ohm fino a un certo valore, raggiunto il quale scendeva rapidamente, e assumeva an regime variabile, a debole intensità. Questo regime una volta raggiunto non si modificava per aumenti o diminuziuni di resistenza, mostrando cosi che von ì indifferente chiudere il circuito su una resistenza grando o su una resistenza debolissima, che si aumenti durante il passaggio della corrente, poichè per ogni interruttore e per ogni f. e. In. esiste una resistenza limite tale, che per ogni resistenza inferiore a essa si ha il solo regine variabile e per ogni resistenza superiore si ha quello variabile o quello continuo a seconda del modo in cui si è stabilita la corronte. Durante il regimo variabile l'A. ha osservato che non si formavano più numerose bollicine di gas, partenti dal filo di platino, ma si liberavano invece nua ad una in modo regolarissino. La guaina di gas che si forma attorno all' anodo $\theta$ che permette di spiegare il fun\%ionamento dell' interruttore, non si forma, secondo l'A. che al momento in cui avviene il cambiamento di regime. Piazzando una lampada di 110 volts in derivazione, sugli estremi dell' interruttore, ha constatato che non si illuminava quando il regime era continuo: s' illuminava invece al momento in cui il regime cambiava, quando cioè la guaina di gas formatasi introduceva una resistenza supplementare. 
Introducendo, durante il regime variabile, una self-induzione nel circuito coine ad es. il primario di una bobina d'induzione, non scoccava nessuna sointilla apprezzabile agli estremi dell' indotto. Introducendo invece questa self-induzione prima della chiusura, l'A. ha ottenuto un terzo regime, discontinuo e a grande intensità media, dotato di proprietá interessanti. In quest' ultimo caso ha constatato un' influenza notevole dell' interruttore sulla illuminazione delle lampade situate sullo stesso circuito, che apparivano di uno splendore maggiore durante il suo funzionamento.

L'A. infine si propone di tornare sull' argomento e di studiare questa influenza e l'estrema stabilitá del regime variabile a debole intensita.

Fabry Ch., Maci de Lepinay a Perot. Sulla massa del decimetro-cubo d' acqux (pp. 709.712). - Con un metodo rapido e preciso gli A. hanno tracciato le curve di uguali spessori per le tro coppie di facce di un cubo di quar\%o di 0",04 circa di lato, le cui facce non erano perfettamente piane nè parallele. Gli A. indicano anche il metodo tenuto per ottenere il parallelismo di queste facce.

Da queste misure differenziali e da misure assolute hanno dedotto il volume del cubo di quarzo, e tenendo conto della massa di acqua a $4^{0}$ spostata e della densità, hanno concluso:

Massa di $1000 \mathrm{cc}$ a $4^{0}: 999^{\mathrm{rr}}, 9786=1^{\mathrm{kgr}}-21^{\mathrm{mr}}, 4$.

Berget A. Registrazione microfonica dell' andamento dei cronometri (pp. 712-713). - L', apparecchio costruito dall'A. per ottenere la trasmissione microfouica dei battimenti dello scappamento consiste in un microfono di Hughes, a carbone verticale a supporto leggerissimo. Il microfono posato semplicemente sul crono. metro da studiarsi, è montato in serie con una pila e un telefono, sulla lastra vibrante del quale è montato un trasmettitore microfonico a quattro carboni. Questo trasmettitore è montato in serie con un ricevitore telefonico, la cui membrana, grairo a una corrente fornita da quattro elementi di pile secche, eseguisce dello vibrazioni di un'ampiezza sufficiente a rompere, a ciascuna vibrazione il contatto fra una punta di carbone e una larnina di platino fissata alla membrana. La rotlura e il ristabilinento di questo contatto, sono atilizzati per produrre su un cilindro rotante annerito l'inscrizione di ciascun battimento prodotto dallo scappamento del cronometro.

L'A. enumera in fine $i$ numerosi vantaggi che ha questo metodo, altre quello di sopprimere l' errore personale di osservazione.

Curif P. - M. Sulla radioattivita provocata dai raggi di Becquerel (pp. 714-716). - Gli A. hanno constatato che i raggi 
emessi da materie fortemente radio-attive, agendo su sostanze inattive possono comunicare loro la radio-attività, che persiste in essi un tempo assai lungo. Per esperienze fatte con sostanze da 万000 a 50000 volte pir attive dell' uranio, gii $\mathrm{A}$. hanno trovato che le attività indotte, immediatamente dopo l'esposizione, variavano fra 1 e 50 volte quella dell' uranio, per ridursi al decimo del loro valore due o tre ore dopo che la sostanza impressionante aveva cessato di agire.

Esperienze ulteriori tendenti a ricercare se la radio-attivitá indotta non fosse dovuta a tracce di materia radio-attiva traspoltate sotto forma di vapore o di polvere sulla lamina esposta, hanno condotto gli A. ad escludere tale spiegazione del fonomeno, o ad anmettere piuttosto che esso si debba a una sorta di raggiamento secondario dovuto ai raggi di Becquerel. Questo fenomeno differisce da quello voto per $\mathrm{i}$ raggi Röntgen, perché $\mathrm{i}$ raggi secondari di questi hanno origiue appena che il corpo che li enctte è colpito dai raggi Röntgen, e cessano bruscainente con essi.

DemarCay E. Sullo spettro del radio (pp. 716-717). — Dal fatto che coll' aumentare del potere radiante del cloruro di bario, aumenta d'intensità la nuova riga segnalata dallo stesso A. nello spettro di quel corpo ') e che ad essa si aggiungono nuove righe, l'A. é indotto a confermare la supposizione che quello spottro sia dovuto alla sostanza radiante. Fotografando lo spettro fra le righo $\lambda=5000,0$ e $\lambda=3500,0$ del cloraro di bario, il cui potere radiante era di 70000 volte quello dell' uranio, ha ottenuto lo spettro del bario completo e intensissimo, quello degli elettrodi a delle impurita debolissino e ridotto alle righe principali, $\theta$ insiene una serie di nuove righe, di cui ha determinato la lunghezza d' onda corrispondente e ha, indicato con numeri l'inteusità.

Heen (DE) P. Riproduzione elettrica delle figure di Savart, ottenute per mezzo di lamine liquide (pp. 717-719). - Disponendo intorno a un piatto di resina elettrizzato dei centri di scuotimento dell' etere, come fiamme o pennacchi elettrici emananti da punte in comanicazione con una bobina, !'A. ha osservato che le cose procedono esattamente come se l'energia elettrica, al pari di un soffio, fosse cacciata da ciascuna di queste sorgenti. Le figure secondo cui si disponeva l'elettricità erano quelle note che si realizzano proiettando su un piano dei getti liquidi.

$\mathrm{Da}$ queste esperienze l'A. deduce che il fenomeno avviene come se quelle sorgenti emettessero un soffio etereo (suffle éthéré) che, incontrando le lanine di resina, vi trasportasse l'energia elettrica, al pari della polvere che il vento trasporta.

1) C. R. 26 Dicembre $1898_{\text {; }}$ 
Lo stesso fatto, secondo l'A., avviene per i raggi $X$, solo che per tali raggi la violenza del soffio é tale, che attraversa la lamina senza distendervisi.

Esperienze nlteriori portano l'A. a concludere per una stretta analogia fra $i$ raggi $X$ e raggi catodici: questi corrisponderebbero a nua proiezione di etere trascinante materia radiante, quelli sarebbero semplicemente proiezioni di etere.

Sariag G. Nuovo modo di considerare la propagazione delle vibrazioni luminose attraverso la materic (pp. 756-758). - In questa Nota l'A. espone un meccanismo fier spiegare la propagazione delle vibrazioni luminose sopratutto cinematico, senza introdurro considerazioni sia dinamiche, sia elettromagnetiche se non quando la natura del problema lo esige assolutamente. L'A. partendo dai principi esposti in questa Nota si propono di trattare in altre Note alcuni problemi, legati finora a considerazioni complesse, in modo semplice e preciso, e di arrivare mediaute essi a risultati nuovi e interessanti.

GAUD F. Sulla spettro-fotomelria delle luci elettriche (pp. 759 760). - Lo studio spettro-fotometrico delle luci elettriche a in. candescenza $\theta$ ad arco è stato fatto dall'A. decomponendo ciascuva di queste luci per mez\%o di scherini di vetro di colori omogenei, e apprezzado l'inteusita del fascio trasmesso per mezzo di un semplice apparectio fotometrico Foucault o Bunsen. Determinata la lunghezza d'onda della radiazione di ciascun schermo, e disposte le sorgenti da studiarsi, munite del vetro giallo, sul fotounetro in modo da ottenere nguaglianza d'illuminazione per la riga $D$, ha sostituito allo schermo giallo successivamente tutti gli altri, variando opportunamente le distanze, per modo da mantenere ugunglianza di illaminazione. $\mathrm{Ha}$ ottenuto in tal modo i rapporti totometrici delle due luci per ciascun colore, che, presi come ordiuate, mentre le ascisse erano le lunghezze d'onda corrispondenti ai colori, hanno dato una curva rappresentante la misura esatta dei rapporti per le righe principali dello spettro, che l'A. riporta in fine della Nota.

Bergler E. Su una nuova lente binoculare (pp. 821-823). L'A. descrive un sistema binoculare destinato a sostituire la lente monoculare, adoperata da molti artisti (orologiai, incisori, ecc.) che non pú rimpiazarsi con microscopi binocularj a causa del. l'ingraudimento tróppo forte che questi producono e della grande piccolezza del loro campo visuale.

Drissiud. Sul rendimento della trasmissione del suono per mezzo dell' elettricitì (pp. 880-881). - L'A. ha constatato un rendimento di gran lunga maggiore di quelli ottenuti fin qui, adot. 
tando come apparecchio trasmettitore una serie di membrane microfoniche chiuse in una cassa di risuonanza, dove l'aria messa in movimento dalla voce era fatta agire su ciascuna dello due facce di queste membrane, che sono riunite da doppi coni e da granuli di carbone. ' L' apparecchio ricevitore era reso più sensibile col dare più faccette ai poli dell' elettrocalamita, dinanzi a ciasciuna delle quali era posta una lastra vibrante, dalle due parti della quale, per mezzo di condotti terminanti a uno stesso orifizio, veniva raccolta l'aria messa in movimento.

Con un apparecchio così costruito l'A. La ottenuto una trasmissione sufficiente per azionare un fonografo e per udire discorsi, ecc. Il rendimento era tale che il timbro e l'intensita della voce erano quasi completamente conservati.

Villard P. Sull' azione chimica dei raggi X (pp. 882-883). Per ricercare se la colorazione violetta che si ha nella parte colpita dai raggi catodici in un tubo di Crookes, che abbia funzionato un po'di tempo, sia dovata ai raggi $X$ o ai raggi catodici diffusi, l'A. ha esperimentato con un tubo focus, il cui anticatodo era circondato da un largo tubo di vetro o di cristallo, la cui parete interna poteva essere protetta contro i raggi catodici per mezzo di una sottilissima foglia di alluminio.

Ha cosi trovato che quando questa foglia noi vi era aveniva solo una riduzione del cristallo, simile a quella che si produce in una fiamma riduttrice, mentre si aveva una cololazione violetta, senza riduzione alcuna, quando si arrestavano i raggi catodici. Avendo inoltre constatato che interponendo sul tragitlo dei raggi $X$ una piccola lamina di platino, la regioue protetta rimane incolora, l'A. no conclude che quella trasfolmazione è dovuta ai raggi $X$, e che $\dot{\theta}$ probabilmente un fenomeno di ossidazione ottenendosi ugualmence collo scaldare il cristallo in una fiamma ossidante. L'A. inoltre pensa che si debba al manganése la colorazione violetta.

BeCQLenel H. Ricerche sui fenomeni di fosforescenza prodotti dal raggiamento del radio (pp: 912-917). - In questa Nota l'A. espone i risultati dei suoi studi sull'azione dell'irraggiamento dol cloruro di bario radifero su diverse sostanze fosforescenti. Ha constatato che quello sostanze, il cui spettro di eccitazione $\dot{e}$ formato da raggi luminosi, non diventano fosforescenti, inentre lo diventano quelle che s'illuminano sotto l'azione dei raggi ultravioletti e sotto quella dei raggi Röutgen.

Pel mezzo di una disposizione fotometrica ha ricercato le in. teusità relative degli effetti luminosi sulle diverse sostanze poste a circa $6 \mathrm{~m}$. dalla materia attiva. Variando questa distanza, ha 
trovato che l'intensita varia un po' piu rapidamente della ragione inversa del quadrato, ciò cho indica un assorbimento per parte dell' aria, che ha riscontrato variabile a seconda dell' irraggiamento particolare che eccita la sostanza stessa. Avendo inoltro os servato che la fosforescenza prodotta è disugualmente indebolita per l'interposizione di schermi di diversa natura fra la sorgente raggiante e le sostanze fosforescenti, l'A. é indotto ad ammettere che ciascuna di esse è eccitata da radiazioni particolari, di natura diversa, e che, caratterizzate dal loro assorbimento, si trovano nella radiazione emessa dalla sorgente, analoghe alle radiazioni di diversa lugghezza d'onda di un fascio dì luce bianca.

Ha rilevato ancora la proprietá presentata da alcuni minerali, e in ispeciale modo dalla fluorina, di conservare per lungo tempo la fosforescenza eccitata in essi dal radio.

Ha del pari constatato che, sotto l' influen 2 a del radio, frammenti di quel minerale calcinati e resi inattivi acquistavano, riscaldati, la proprieta di dare una luce identica a quella che danno nelle stesse condizioni quando sia fatta scoccare in loro prossimità una scintilla elettrica.

Ha infine rilevato l'indipendenza fra gli effetti di fosforescenza e il fenomeno, scoperto dal Curie, di potere temporanea. mente rendere l'aria conduttrice per parte dei corpi sottoposti all' influenza del radio. L' esperieuze fatte hanno provato, secondo l'A., la realta di una emissione continua di energia per parte dei corpi radio-attivi, contenente radiazioni diverse, $\theta$ presentanti fra loro differenze del medesimo ordine di quelle obo passano fra le radiazioni luminose di diversa lunghezza d'onda e $i$ raggi $X$ socondari del Sagnac.

Perreau. Influenza dei raggi $X$ sulla resistenza elettrica del selenio (pp. 956-957). - L'A. studiando se e come variava là resistenza del selenio sotto l'azione dei raggi $X$, ha trovato che questi raggi producono su quella resistenza lo stesso effetto dei raggi luminosi. Una resistenza che nell' oscurita era di 40000 ohms, sotto l'azione dei raggi $X$ scendeva rapidamente a 34000 ohms, per ritornare al suo valore primitivo appena sottratta dall'influenza di que' raggi. L'A. ha provato anche se le vibrazioni erziane modificavano del pari la resistenza del selenio, ma ha ottenuto un risultato negativo.

Tommasina T. Sulla constatazione della fluorescenza dell'alluminio e del magnesio nell' acqua e nell' alcool solto l'azione delle correnti della bobina d'induzione (pp. 957-959). - L'A. adoperando un tubo ovoide di Crookes riempito di acqua distillata e di cui l'anodo era costituito da due specchi concavi di alluminio $\theta$ il 
catodo da un filo di platino, ha osservato che la fluorescenza dei due specchi aumentava coll' intensità della corrente. Un filo di atnmonio sostituito al filo di platino diveniva luminoso insieme coi dischi, e, al pari di questi, si presentava coperto di numerose bollicine gassose con piccolissime suintille. Ha inoltro constatato che l'azione di questa luminositá è alternativa, e che la corrente di apertura rende luminoso l'anodo, quella di chiusura il catodo.

Variando i metalli ha trovato che solo l'alluminio $e$, in grado minore,'il magnesio diventavano luminosi, per i quali corpi la fluorescenza aumentava e diveniva più bianca coll' aumentare l'intensità della corrente e col diminuire la superficie degli elettrodi.

Disponendo poi una lamina di alluminio fra due lamine di platino fissate in un voltametro, ha trovato che essa s' illuminava da una sola parte o da entrambe a seconda che le due lamine oostituivano entrambe il catodo, funzionando essa da anodo, o una sola di esse fupzionava da anodo mentre l'altra costituiva il vato do.

Da queste esperienze l'A. ha potuto rilevare cho la fluore. scenza si manifesta piu rapidamente sulle lamine già adoperate o ricoperte da un leggero stato di ossido.

Per i liquidi l'A. ha trovato che il fenomeno si manifesta in modo migliore nell' acqua distiklata e nell' alcool, e un po' meno nell' acqua ordinaria e nell' acqua contenente qualche goccia di acido solforico: non si manifesta negli olii dielettrici.

L'A. infinu crede che il fenomeno si produca durante l'elettrolisi per scariche successive tra il metallo e il liquido attraverso il minuto strato dielettrico formato dall'ossido. La fluorescenza quindi avrebbe la sua causa diretta del flusso elettrico attraverso l'ossido.

Becquarel $H$. Influenza di un campo magnetico sul raggiamento dei corpi radio attivi (pp. 996.1001). - Il campo magnetico adoperato dall'A. era formato da dae piccole superfici di ferro dolce, piane e circolari, che si potevano calatnitare fino a saturazione per inezzo di una forte elettrocalamita. Per studiare gli effetti prodotti nel senso delle linee di forza, la materia radiografica, coperta di una minuta foglia di alluminio, era disposta presso il centro di una delle superfici polari, mentre contro l' altro polo ora disposto un quadro fluorescente. Finchè l'elettrocalamita non era eccitata, la fosforescenza appariva debole, sotto forma di una macchia luminosa, estendentesi al di là della superficio polare. Eccitando l' elettrocalamita la macchia diveniva piủ intensa, il bagliore sorpassava appena i contorni della regione polare e tutti i raggi eccitatori emessi dal radio, sembravano cadore sn questa 
superficie. L'jntensitá non cambiava al cambiare del senso di magnetizzazione.

In un piano perpendicolare al campo, quando la materia attiva era posta nol mezzo del campo, e i punti dello suhermo fluorescente, formato da un cilindro il cui asse parallelo passava per la sorgente ragginte, erano sensibilmente alla medesina distnnza dalla sorgente e in una direzione normale al cainpo, si aveva una diminuzione considerevole nella fosforescenza, che si riduceva appena percettibile eccitando l' elettrocalamita.'

Piazzando poi la materia attiva al disotlo della patte più intensa del campo, e uno schermo fluorescente al disopra dalla parte opposta, si aveva una diminuzione o un auneato nella fosforescenza a seconda del senso della corrente.

Esploraudo infine il campo per mezzo della fotografia, l' A. ha oltenuto curve che presentano il massino d'impressione in punti diversi a seconda della posizione occupata dalla nateria attiva.

L'A. couclude che il raggiamento del radio si riavicina considerevolmente ai ruggi catodici, e che certe esperienze danno la riproducione quasi esatta delle esperienze eseguite sui raggi catodici dal Broca ').

Gerschus A. Metodo per determin bre la densità media della terra e la costante di gravituzione (pp. 1013-1015). - In questa Nota l'A. dà la descrizione schematica di un nuovo metodo per determinare la densita inedia della terra e la costante di gravi. tazione, e $\mathrm{i}$ cui dettagli annunzia cho saranno pubblicati nel Journal de la Societé astronomique russe.

BecQurrer. H. Sulle radiczioni dei corpi radio-attivi (pp. 12051207). - L'A. dichiara che studi ulteriori relativi all' azione di un campo unguetico sulle radiazioui dei corpi radio-attivi, gli hanno mostrato che questa azjone non sembra essere generale, poiché la radiaziono di una preparazione di azotato di polonio, piazata in un campo magnetico, non ne ha risentito alouna influenza, meutre l' ha risentita il radio posto nelle stesse condizioni.

Proseguendo le ricerche descritte nella sua ultima Nota ha constatato juoltre che le ralliazioni del radio, pure approssimandosi considerevolmenle ai raggi catodici, non sembrano possederne tutte le proprietà.

Per mezzo di esperienze eseguite in un campo magnetico l'A. ha trovato che la velocita di propagazione delle radiazioni del

1) Jouru, ds Phys., 3. serie, t. 7, p. 710, 1898. 
radio è dell' ordine di grandezza della velocitá di rotazione comunicata da un campo magnetico di 4000 unità C. G. S.

L'A. annunzia infine che pubblicherà quanto prima le misure dei rapporti tra la velocita di propagazione e la velocità angolare d'impulso del raggiamento.

Delezinier. Dell' impiego delle correnti trifasiche in radiografia (pp. 1227-1228). - Dalle esperienze eseguite dall'A. sull' impiego delle correnti trifasiche nella radiogratia risulta obe mentre nessur tubo piazzato sull' indotto si rischiara eccitando la bobina per una presa di corrente su due qualunque dei fili di una trifase, e clo gli interiultori diversi messi nel primario o non funzionauo, o, come il Wehnelt, funzionano in modo irregolare, si possono ottenere ottimi effecti interponendo un elettro a nucleo fogliato, formato da due brauche rettilinee a $120^{1}$, portante due bobine di senso inverso, di debole resistenza, di cui il numero delle spire decrescono di due giri per strato. Fra gli interruttori il più soddisfacente $\dot{\theta}$ il solenoide verticale a estremità inferiore inmersa nel mercurio.

L'A. ha potuto in tal modo ottenere, con un materiale ordinario per correnti continue, una serie di ben riuscite radiografie.

GaLY-Achì. Su alcuni fenomeni che presenta il ferro (pp. 1230-1232). - L'A. ha esperimentato su cilindri di ferro pressochè paro chimicamente, alti $13 \mathrm{~mm}$.

Comprimendoli ha trovato che ciascuno di essi si sohiacciava di virca $0^{10}$ "10 sotto la pressione costante di $900 \mathrm{kgr}$, corrispondente a $18 \mathrm{kgr}$. per $\mathrm{mm}^{2}$. Lo schiacciamento cresceva in seguito colla pressione, e se, togliendo questa pressione, tornava ad eser. citarla progressivamente, non si avova alcun effetto finchè essa non aveva riacquistato il valore primitivo. Aspettando ad esercitarla di nuovo dopo qualche ora, il cilindro poteva sopportare una pressione superiore alla pressione anteriormente esercitata, senza dare segno di schiaccianento. L' elevamento del limite elastico cresceva col tempo e tendeva verso un limite.

I'A. ha studiato pure l' influenza della velocità di raffredda. monto su questi cilindri, scaldandoli fino alla temperatura di $850^{\prime \prime}$, e lascinndoli raffreddare lentamente o bruscamente. Sottoposti alla cumpressione ha trovato che nel $2^{0}$ caso non presentavano più il fenoneno di sosta (palier) che avveniva invece nel primo caso. Questo fenomeno riappariva col tempo, scaldando leggermente $i$ cilindri, e sussisteva sempre qualunque fosse la velocita di raffreddamento, se la temperatura a cui erano portati era inferiore di $850^{\circ}$. 
L'A. ha notato infine che fino alla temperatura di $1000^{\circ}$ il limite elastico sembrava indipendente dalla temperatura a cui era stato portato il ferro e dalla velocita di raffreddamento. Per una temperatura saperiore a $1000^{\prime \prime}$, il limite elastico del ferro raffreddato brascamente, diminuiva colla temperatura.

Questi fatti portano l'A. a concludere, con Osmond e Werth, che esistono due varietà allotropiche del ferro: una stabile alla temperatura ordinaria, l'altra stabile ad alte temperature. Questa seconda varietà puó ottenersi a temperatura ordinaria sia per raffreddamento brusco, sia per deformazione del inetallo. $\mathrm{Ma}$ il ferro nel secondo stato passa al primo stato lentamente alla temperatura ordinaria, rapidamente se si eleva la temperatura manteuendola al disotto del punto di trasformazione.

\section{P. BACCEI.}

\section{Journal de Physique.}

T. VIII, 3. serie, $2^{\jmath}$ sem. 1899.

Amagat H. Su una nuova forma della relazione $f(p, v, t)=0$ relativa ai fluidi (pp. 353-362). -- L'A. avendo notato che la forma della funzione $f(p, v, t)=0$ adottata in un precedente lavoro '), mentre rappresenta con notevole esattezza $i$ dati relativi all'idrogeno fino verso 3000 atm. tra $0^{\prime \prime}$ e $200^{3}$, non porta nessun buon risultato per l'acido carbonico, determina in questa Nota la forma di una nuova funzione $t^{\prime}$ da sostituirsi a $\mathbf{P}$ nella relazione

$$
(p+\mathrm{P})(v-a)=\mathrm{RT}
$$

in luogo della funzione $\pi=\mathrm{T} \frac{d p}{d t}-p$ sostituita a $\mathrm{P}$ stesso nel lavoro citato.

Tenendo conto delle condizioni cui deve soddisfare $\pi^{\prime}$ di crescere, a una data temperatura, colla pressione, di passare per un massimo, di diminuire, di annullarsi $\theta$ di assumere $\nabla a l o r i$ negativi rapidamente, crescenti e, per un volume dat $\sigma$, di diminuire all' aumentare della temperatura, dopo calcoli che l'A. stesso dichiara lunghi e laboriosi, trova per $\pi^{\prime}$ la forma:

$$
\frac{v-\left[a+m(v-b)+\frac{c}{v-b}\right] \mathrm{T}}{k v^{288^{5}}-x+n v \overline{(v-\beta)^{2}+d^{2}}}
$$

1) Journ. de Phys. t. 3, 3. serie, pag. 387, 1894. 
- la relazione (1) diventa

$$
\left\{p+\frac{v-\left[a+m(v-b)+\frac{c}{v-b}\right] \mathrm{T}}{\left.k v^{3 / 35}-x+n V v-\beta\right)^{2}+d^{i}}\right\} v=\mathrm{RT}
$$

dove I é la temperatura assoluta, e le costanti hanno i valori seguenti :

$a=0,0000014566 ; \quad b=0,000947 ; c=0,0000000028832 ; \quad m=0,0018$; $\mathrm{R}=0,00368 ; k=44,6 ; x=0,000000198 ; \beta=0,0018425 ; d=0,0802679$;

$$
n=0,0006 \text {. }
$$

L'A. riportando quindi in una tavola $i$ risultati delle sue esperienze su una unassa di acido carbonico occupante un volume uguale all' unità a $0^{\prime}$ e sotto la pressione normale, nota che le pressioni necessarie per mantenere il gas sotto d eterminati volumi, a diverse temperature, ottenute dalle esperienze sono assai concordanti con quelle calcolate per mezzo della formula, mostrando soltanto divergenza alle temperature più alte.

L'A. passa pure a ricercare con quale grado di approssimazione la fanzione da lui determinata puó prestarsi al calcolo dei dati relativi alla saturazione, e trova risultati assai soddisfacenti, date le difficolta delle esperienze di simile genere. Le tensioni sperimentali sono generalmente comprese tra le pressioni calcolate $\theta$ pochissimo diverse dalla loro media.

L'A. infine dopo avere rilevato come la formula andrebbe modificata perchè fosse rigorosamente esatta, conclude che per arrivare a buoni risultati occorre proseguire lo studio sperimentale delle isoterme sotto pressioni sufficientemente duboli, restaudo nei limiti fra $i$ quali gli errori relativi che comporta la loro misura, non cominciano a divenire notevoli.

Bıoxdot R. Produzione di forze elettromotrici per lo spostamento in seno a un liquido, sottoposto all' azione magnetica, di masse di conduttività differenti (pp. 362-366). - Il fenomeno che forma oggetto della preseute Nota è stato studiato dall'A. ponendo na vaso di forma parallelepipeda rettangolo alto 0 n, 23 , largo $0^{m}, 18$ nel campo di un'elettrocalamita, i cui pezzi polari erano formati da due larghi piatti, in modo che le linee di forza lo traversassero nornalmeute al suo spessore. Il vaso conteneva al suo fondo per circa 00,03 di altezza una soluzione concentrata di solfato di zinco, al disopra della quale era versata con cura, per impedire quanto più era possibilo il miscuglio, altra soluziono diluitissima 
del medesimo sale. Su due facce opposte, all' altezza di 0"1,04 dal fondo, erano praticati due fori, ai quali erano masticati due tubi ricurvi verso il basso e chiusi da un diaframıa di cartapecora: ciascuno di questi tubi pescava in un bicchiere contenente una stessa soluzione di solfato di zinco e munito ai un elettrodo di zinco amalgamato. I due elettrodi erano collegati agli estromi di un elettrometro capillare, che non dava nessuna indicazione finchè i liquidi erano in quiete. Agitandoli invece con una bacchetta di ebanite in modo che avvenisse il miscuglio delle due soluzioni, senza che l'agitazione raggiungesse la superficie libera del liquido, l'elettrometro indicava una differenza di potenziale agli eletcrodi, che spariva appena si cessava di provocare il miscuglio per riapparire di nuovo mettendo in azione l'agitatore, $\theta$ spariva definitivamente quando il miscuglio era completamente avvenuto.

Invertendo jl campo magnetico o annullandolo si cambiava di senso o si anuullava anche la differenza di potenziale. Il senso di questa differenza era dato dalla seguente regola: un individuo coricato nel vaso, sul fianco sinistro e col viso volto verso il polo australe della calawita, ha l' elettrodo positivo dal lato della saa testa.

Analizzando il fenomeno l'A. conclude che esso è dovuto all' induzione che tende a produrre uno spostamento di elettricità positiva in un senso della larghezźc $\theta$ di eletricità uegativa nel senso opposto. Sul principio dell' agitazione del liquido quelli spostamenti hanno luogo senza ostacolo; pui l' accumulazione delle cariche che ne risulta dà origine a forze elettriche, opposte alle forze elettromotrici $d$ 'induzione, e ne diminuiscono gli effetti in seno al liquido. Queste stesse cariche, d'altra parte, elevano il potenzialo verso un elettrodo e lo diminuiscono verso l'altro.

L'A. quindi mostra che il fenomeno obbedisce a una legge analoga a quella di Lenz, e pensa che esso debba avvenire anche quando si lasciano diffondere $i$ due liquidi spontaneamente l' uno nell' altro nol cainpo magnetico, pur dubitando che in questo caso, data la sua piccolezza, riesca facile o possibile l'osservarlo. Crede invece che il fenomeno avvenga e acquisti una iutensità notevole in seno al mare, e più specialmente alla foce dei fiumi per il miscuglio di acque di salsedine diversa nel campo magne. tico terrestre.

Descrive infine un' altra esperienza analoga nella sostanza, se non nella forma, a quella già indicata, $\theta$ comprovante la grande conduttibilità del rame rapporto al mercurio. Poneva nel campo magnetico un vaso di legno, di forma e dimensioni uguale a quello di vetro precedentemente adoperato, contenente mercurio. In con- 
tatto col mercurio poneva due fili di rame masticati a due facce opposte del vaso e collegati con un elettrometro. Faoendo quindi salire o discendere in seno al mercurio una sbarra di rame rosso, sostenuta da un'asta isolaute, coustatava a ogni movimento una forte deviazione all' elettrometro il cui senso indicava che il flusso di elettricità prodotto dall' indusione era più grande attraverso il rame che attraverso il mercurio. Lo stesso risultato aveva facendo salire o discendere simultaneamonte nel inercurio masse di rame di forma qualunque e separate le rine dalle altre.

Potrevin H. Contribuzione allo studio del potere rotatorio molecolare dei corpi disciolti (pp. 373-376). - Pribram ${ }^{1}$ ) ha indicato che il potere rotatorio delle soluzioni acquose di acido tartarico va diminuevdo coll' agginggere loro quantità crescenti di alcool (alcool metilico, etilico, propilico) o d'acido grasso (acido formico, acetico); l'A. avendo constatato che l'aldeide formica agisce inversamente dell' acido e dell' alcool corrispondente, si $\dot{e}$ proposto di studiare questa particolaritá e di vedere se modificazioni analoghe di potere rotatorio si producessero con altre sostanze attife e con altre aldeidi.

Le rotazioni sqno state riportate alla luce gialla del sodio e le misure sono state fatte alla temperatura da $20^{\prime \prime}$ a $22^{3}$.

$\mathrm{Ha}$ trovato cosi che l'aldeide formica aumeata la rotazione delle soluzioni di tartarati, modifica pure nel senso destrogiro i poteri rotatori dell'alcool amilico, dell' essen\%a di matricaria e non ha alcuna azione sull' essenza di trementina.

Per l' acido tartarico è arrivato alle conclusioni :

I. Le aldeidi formica ed etilica si comportano inversamente degli alcool e acidi corrispondenti.

II. L' accrescimento del potere rotatorio dipende non dalle proporzioni relative di aldeins e di acido, ma dalle proporzioni relative di aldeide $\theta$ di acqua.

LipPManN G. Sulla misura assoluta del tempo dedotta dalla legge dell' attrazione universale (pp. 401-407). - L'A. detto che cosa s'intenda per misura assoluta, passa a ricercare la misura assolnta del tempo, non essendone il secondo che una misura ar. bitraria.

Prese l' unità di massa, l' unità di volume e l'unità di densità quali ordinariamente si prendono, stabilisce l' uguaglianza fra le due espressioni $K^{2} \frac{M m^{\prime}}{r^{2}}$ e $m \frac{\partial^{*} x}{\partial t^{z}}$ che danno la forza Fesercitantesi tra due masse $M, m$ separate dalla distanza $r$, e uelle 
quali $k$ rappresenta la costante newtoniana, $\frac{\partial^{2} x}{\partial t^{2}}$ l'accelerazione o $t$ il tempo espresso in funzione di an' uaita qualunque. Trova cosi che $k$ e indipendente dalle anita di lunghezza $\theta$ di massa, $\theta$ che il suo valore numerico é proporzionale alla grandezza dell' unitá di tempo. Ne risulta che esiste solo un intervallo di tempo e uno solo, preadendo per nnità il quale, si ha $\mathbf{K}=1$. Supponendo adottata questa unita e chiamando $\theta$ il tempo cosi misurato, ha per $\mathbf{F}$ l'equazioni:

$$
\mathrm{F}=\frac{\mathrm{M} m}{r^{-2}} \quad \text { (2) } \quad \mathrm{F}=m \frac{\partial^{2} x}{\partial \hat{\theta}^{2}}
$$

che non contengono $k$. Fra $t$ e $\theta$ pazsa poi la relazione:

$$
k t=9
$$

che esprime che $k$ è il valore numerico dell' antica unità in funzione dell' unita che dà per la costante newtoniana il valore numerico 1. L'A., per analogia con quanto si ha per $i$ logaritmi volgari, propone di chiamare ora naturale l'intervallo di tempo che preso per unith, fornisce il sistema delle equazioni (1) e (2).

Per mostrare che si possono dare più definizioni fisiche di questa unità, applicando la sua definizione analitica a una serie di casi particolari, studia il caso di un punto materiale gravitante attorno a una massa $M$ descrivente un' orbita circolare, e quello delle oscillazioni di un pendolo di lunghezza ridotta.

Indica pure sommariamente come si puó determinare il valore di $k$ relativo al secondo, calcolando la durata di un movimento dovato all' attrazione newtoniana, le oscillazioni di un pendolo, ecc.

Conclude infine che per misurare il tempo in valore assoluto, basta prendere la costante dell' attraziono newtoniana uguale a 1 . L' unità di tempo allora messa in uso è completanente determinata, indipendente dalla scelta dell' unità di lunghezza, ed espressa in tempo medio da 3862 secondi, pari a $1^{\text {lh }}, 4$ "', 22 s.

Le equazioni (1) e (2) determinano, oltre l'unità di tempo, l'unità di forza, la quale dipende dalla scelta dell' unità di lunghezza, ed è uguale all' attrazione dell' unità di massa sull' unità di massa all' unità di distanza, o, puó dirsi, è uguale al peso dell' unità di-massa alla superficie di un pianeta che avesse l'unità di massa e l'unità di rággio.

Osserva infine che, facendo uso del sistema precedente, lo quantità elettrostatiche avrebbero formule di dimensioni semplicissime: la massa, il potenziale, il campo ecc. elettrostatici avreh- 
bero le stesse dimensioni della massa, del potenziale e del campo newtoniano.

Caliro J. Misure sul microfono (pp. 413-416). - Il microfono si trovava in un circuito comprendente una pila e il primario di una bobina. Pe! un'onda sonora che colpiva la lamina del microfono, si produceva una variazione nella resistenza dei contatti microfonici e quindi una variazione della corrente primaria, alla quale corrispondera nel secondario della bobina una f. e. $m$. che produceva una corrente alternata nel circuito del secondario. Il secondario era costituito dalla bobina, dai telefoni di partenza, dalla linea, dai telefoni e da una bobina all' arrivo.

L'A. ha misurato la variazione media dell' intensitá della corrente, la porzione efficace della variazione alternata nel cilcuito primario e nel secondario, la $f$. $\theta . \mathrm{m}$. indotta nella bobina in circuito aperto, l' intensitá I della corrente che aziona i telefoni all' arrivo, la differenza di potenziale efficace agli estremi del telefono ricevitore e l'energia elettrica assorbita nel telofono. $\mathrm{Ha}$ infine ricereato come queste quantità variavano col periodo e coll' ampiezza del suono.

Limitandosi allo studio dei suoni musicali, ha trovato che:

Ia frazione efficace della variazione alternata dell' intensita della corrente primaria $\dot{e}$ indipendente da questa ed $\dot{\theta}$ inferiore a 'is per i suoni più forti. Essa è press' a poco proporzionale all'ampiezza della vibrazione sunora che aziona l'apparecchio e non sembra dipendere dall' altezza del snono: la variazione continua è molto più debole.

La f. e. m. efficace in circuito aperto nel secondario è di circa 1 ', $\mathbf{5}$ per $i$ snoni più forti : la f. e. m. massima corrispondente è di $2^{v}, 2$. Essa varia press'a poco come l'ampiezza della vibrazione agente, $\theta$ in ragiono inversa del periodo.

Nel oaso del suono più forte la differenza di potenziale agli estremi del telefono é espressa da centesini di volts, e l'energia assorbita da milionesini di watt.

L' jntensità efficace è dell' ordine dei centomillesicui di am. père e discende fino ai milionesimi di ampère per suoni ancora nettamente percettibili. $\dot{\mathbf{E}}$ sensibilmente proporzionale all'ampiezza dell' onda sonora e non seubra variare col periodo, alla quale legge deve quindi obbedire lo spostamento pure della membrana telefonica.

Da questi risultati l'A. trae la conclusione che i suoni non devono venire modificati in modo notevole dal telefono, $\theta$ che quindi il timbro non deve essere troppo alterato nel caso di un suono musicale complesso. 
Gadtin P. Sul grande siderostato del 1900 (pp. 417-431'. -In questa Nota l'A. dà varie, iuteressanti notizie sul graode siderostato che figurerá all' Esposizione di Parigi di quest'anno, accenuando alle molteplici e grandi difficolta superate per la sua costruzione e installazione. Per ottenere una maggiore stabilita e per evitare al tempo stesso ohe l'osservatore dovesse continuamente spostarsi è stato adottato il siderostato di Foucault, la cui montatura offre, oltre quelli detti, vari altri vantaggi come quello di mantenere il cannocchiale sempre nella medesima posizione, di rendere l'obbiettivo meno suscettibile di flessione e di rendere infine meno volumivosa la parte mobile. La Nota tratta quindi della teoria dello spostamento dello specchio dal siderostato, del suo meccanisino, del modo di regolare l'istrumento e del modo di manovrarlo.

11 corpo dell' istrumento è lungo $60 \mathrm{~m}$. e ha $1^{\prime \prime \prime}, 50$ di diametro. $\dot{E}$ formato da 24 tubi di $2 \mathrm{~mm}$. di spessore. $\dot{E}$ fissato $\theta$ riposa su cinque punte d'appoggio della stessa altezza, per modo che il suo asse si trova al livello del centro dello specchio. Pesa $21,000 \mathrm{kgr}$, e tutto l' istrutuento ne pesa 38,000 . Gli obbiettivi, uno astronomico e uno fotografico di $1^{\text {"n }}, 25 \mathrm{di}$ diametro, sono co. stituiti da due lenti, una in crown e una in flint; l'insieme di ciascun obbiettivo pesa $900 \mathrm{kgr}$. Le lenti si possono con meccanismo semplice spostare e ripulire. La distanza focale di questi obbiettivi è di $60 \mathrm{~m}$.

Il gruppo dell' oculare è formato da un tubo, che può facilmente spostarsi in guisa da potere mettere a fuoco la lastra fo. tografica o l'oculare che s'impiega. Dentro a questo tubo ne puó scorrere un secondo di m. 1,20 di diametro, che porta il micrometro o la lastra fotografica. Per ottenere l' immobilità del campo rapporto al reticolo, questo secondo tubo riceve un movimento di rotazione, comandato da un movimento di orologeria, che gli viene trasinesso mediante un sistema che lo rende variabile secondo i diversi paralleli, fra limiti estremi constalati esperimentalmente. Altre parti unite all' oculare permettono sia di orientare rapidamente il reticolo, secondo l'inclinazione voluta, per la misura delle distanze fra le stelle, sia di seguire, immabilizzando lo specchio, una stella durante il suo passaggio nel campo del cannocchiale, altre infine di far fare all' oculare movimenti lenti in ascensione retta $\theta$ in declinazione.

Con le disposizioni adottate, secondo l'A., la fissità del canpo all' oculare $\dot{\theta}$ perfettamente assicurata, e lo strumento $\dot{e}$ di una stabilità, di una precisione e di una facilita di manovra, quale 
non si potrebbe trovare negli istrumenti equatoriali di piccola dimonsione.

Nikolateve W. Sulle azioni meccaniche della scarica disruttiva (pp. 432-433). - Per fissare l'effetto della scarica in seno dell' aria l'A. ha fatto uso di uno strato di cotone igroscopico pressato fra due anelli di ebanite $\theta$ interposto fra due elettrodi di una batteria di diciassette grandi bottiglie di Leyda, caricata da una macchina Holtz.

Per studiare il processo della scarica in seno agli elettroliti e ai dielettrici liquidi, ha fatto uso dello stesso strato di cotone imbevato fino a saturazione di acqua potabile o di olio di vasellina.

Ha trovato cosi che nei liquidi si produce lo stesso effetto di esplosione che nell' aria, la quale osservazione serve, secondo l'A., a spiegare come l' effetto dell' esplosione è legato alla distruzione brusca di certi tibi di forza elettrica e al movimento tra. sversale di quelli rimasti intatti.

Ha studiato pure la deformazione e la perforazione delle lastre e delle foglie metalliche, interponendo delle foglie di alluminio fra delle lastre di cartone o inversamente, e piazzandole tra gli elettrodi di una batteria perpendicolarmente alle linee di forza. Ha constatato la formazione nel metallo di cavità, la cui apertura e profondità dipendono dalla intensitł della scarica e dalla distanza del metallo dal cartone, in fondo alle quali l'esame microscopico ha rivelato l' esistenza di gocciolette solidificate, indice questo di una fusione del metallo avvenuta in questa regione durante la scarica. Questi effetti sono stati più notevoli con foglie di rame rosso di $0^{m}, 05$ o $0^{n}, 025$ di spessore, e aurneutano considerevolmeute ricoprendo il metallo di un sottile strato di paraffina.

Da questi risultati e da altri ottenuti formando degli elettrodi con foglie di stagno o seminando su una lastra netallica orizzontale della polvere di Jicopodio e facendo avvenire la scarica fra questa lastra e una punta situata al disopra, l'A. ha concluso che gli effetti della scarica si riassumono in una forte elovazione di temperatura $e$ in una deformazione, forse dovata all'esplosione nell' aria.

Nikolainve W. Sulla produzione della rotazione elettromagnetica degli elettroliti (p. 434). - In un vaso cilindrico di 10 o $16 \mathrm{~cm}$. di diametro, contenente una soluzione di solfato di rame, funzionano da elettrodi due dischi di rame o due anelli metallici, disposti perpendicolarmente all' asse del cilindro, uno in basso e l'altro in alto. Piazzando il vaso cilindrico fra due poli magne- 
tici dello stesso nome, si osserva la rotazione del liquido in un senso o nell' altro.

Haqa H. o Wind C. Diffrazione dei raggi di Röntgen (pp. 434.435j. - - In questa breve Nota G. Sagnac' riferisce un brano di una lettera a lui diretta da H. Haga e Wind C. nella quale essi ribattono le osservazioni da lui fatte alle loro esperienze sulla diffrazione dei raggi Röntgen, $\theta$ mantengono le conclusioni alle quali erano giunti con quelle esperienze, che cios i raggi $X$ formano gruppi di raggi, di cui le lunghezze d'onda variano da

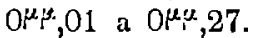

SACERDOTH P. Deformazioni elettriche dei solidi dielettrici isotropi (pp. 457-471). -- Già da lungo tempo si è constatato cho i solidi dielettrici si deformano, quando diventano la sede di campi elettrici, ma i risultati esperimentali erano, su certi punti, in contraddizione gli uni cogli altri e con i risultati teorici, pure discordi fra loro.

L'A. si è proposto quindi:

$1 .^{0}$ di stabilire le formule di deformazione dei dielettrici dei condensatori, basandosi unicamente sui principi fondamentali della conservazione dell' energia e dell' elettricitá, d' indagarne le leggi e di dedurre le cause di quei fenomeni;

2 . $^{\circ}$ di mostrare che tutte le divergenze presentate dalle teorie precedenti erano dovute a errori che, rettificati nna volta, modifica so sempre $i$ risultati in accordo colla sua teoria;

3." di mostrare, facendo la critica dei lavori sperimentali, le canse per le quali essi non hanno sempre confermate le previsioni teoriche.

Premesso quindi uno studio preliminare sulle variazioni che prova la costante dielettrica di un solido prinitivanente isotropo allorchè si deforma meccanicamente, passa alla teoria che applica al caso del condensatore sferico infinitamente sottile, al caso del condensatore cilindrico a al caso del condensatore piano. Trova cosi delle formule che traduce a seconda della loro forma in - leggi delle deformazioni unitarie » e "leggi delle deformazioni .

Delle leggi delle deformazioni unitarie da l'enunciato segruente: Tutte le deformazioni unitarie che subisce il dielettrico sono proporzionali al quadrato dell' intensità del campo elettrico o proporzionali ancora al quadrato del potenziale inversamente proporzionali al quadrato dello spessore del dielettrico, essendovi dci coefficienti (di cui l'A. dà l'espressione) che dipendono unicamente dalla natura del dieleltrico, non avendo alcuna influenza nè la formx, nè la grandezza del condensatore.

Lo leggi delle deformazioni sono: 
1a Ogni linea perpendicolare alle linee di forza prova una variazione di lunghozza proporzionale alla sux lunghezza, al quadrato del polenziale e all' inversa del quadrato dello spessore del dielettrico.

$2^{4}$ Lo spessore del dielettrico (uella direzione del campo) varia proporzionalmente al quadrato del potenziale e all' inversa di questo spessore.

3a Lue cavità provano delle variazioni di volume proporzionali al loro volume, al quadrato del potenziale e all' inversa del. quadrato dello spessore del dielettrico.

4. La materia dielettrica prova una variazione di volume proporzionate alla superficie del condensatore, al quxdruto del potenziale e all' inversa dello spessore del dielettrico, o, dicendo altrimonti, proporzionale all' energin elettric.t del condensutore.

Passando poi al caso in cai il coudensatore sin sempliceunente sottile, anzichè infinitanente soțtile, o di uno spessoro qual unque, l'A. trova che:

Nessuna delle leggi precedenti sussiste, all' infuori di quella relativa alla proporzionalitio tra la grandezza delle deformazioni il quadrato del potenziale; in particolare le differenti linee perpendicolari alle linee di forza subiscono delle dilatezioni unitarie disuguali.

Studiando finaluente il caso in cui le armature sieno indipendenti dal dielettrico, l'A. trova delle formule, nelle quali $i$ termini che contengono $i$ coefficienti elastici sono completamente diversi, in grandezza $e$ in segno, da quelli ottenuti nel caso in cui si ha contatto fra le armature e il dielettrico: al controrio i termini in $\left(k_{1}, k_{2}, k\right)$ restano sompre gli stessi, indicando $k_{1}$ il coefficiente di variazione della costante dielettrica per una trazione perpendicolare alle linee di for $\iota a, k_{3}$ il coefficiente di variazione della stessa costante per trazione parallela alle linee di forza, e $k$ infine il coefficiente di variazione per trazione superficiale uniforme.

Gullew A. Determinazinne diretta di un Kilohm assoluto (pp. 471-477). - Con un metodo analogo a quello descritto nel 1887 da Lippmann nel suo lavoro sulla costruzione di un campione elettrico, l'A. si è proposto di costruire un Kilohm assoluto.

E partito dal principio che il sistená magnetico di un galvanometro Thompson, montato in differeoziale e di cui una coppia di rocchetti, di resistenza $G_{2}$, riceva $n$ scariche per secondo dello stesso valore $q$, mentre l'altra coppia di resistenza 
$G_{1} \dot{e}$ percorso da una corrente compensatrice $i$, è ridotto a zelo, quando sia soddisfatta la relaziono:

$$
n q=i \text {. }
$$

'Tenendo conto che l'induzione è prodotta in un circuito di resistenza $r$, tra due bobine di induzione mutua $\mathrm{M}$, per il ristabilimento o soppressione di una corrente d'intensità $I$, si ha per $q$ il valoreg :

$$
q=\frac{\mathrm{MI}}{r}
$$

e indicando con $V$ la differenza di potenziale fra $i$ punti $\alpha$ e $B$ fra i quali è intercalata la resistenza da valutare, si ba:

$$
\mathrm{I}=-\mathrm{V}
$$

Chiamando poi S una resistenza modificabile che completa la resistenza $\mathrm{R}$ del circuito componsatore, e dai cui estromi partono i fili che vanno alla bobina $G_{1}$, e ponendo:

$$
R^{\prime}=R+\frac{S G_{1}}{S+G_{1}}
$$

si ha:

$$
i=\frac{\mathrm{S}}{\mathrm{S}+\mathrm{G}_{1}} \cdot \frac{\mathrm{V}}{\mathrm{R}^{\prime}}
$$

o quindi l'equazione di equilibrio assume la forma :

$$
\frac{n \mathrm{M}}{r \cdot \mathrm{R}}=\frac{\mathrm{S}}{\mathrm{S}+\mathrm{G}_{1}} \cdot \frac{1}{\mathrm{R}^{\prime}}
$$

dalla quale puó dedursi il valore di $R$.

Questo valore quando si supponga che l'azione di una corrente passante in $G_{1}$ sia colupensata da quella di una corrente $p$ volte più debole passante in $G_{2}$ i dato da:

$$
\mathrm{R}=p n \mathrm{M}\left(1+\frac{\mathrm{G}_{1}}{\mathrm{~S}}\right) \frac{\mathrm{R}^{\prime}}{r}
$$

e poichè invertendo l'ufficio dei due rocchetti, si avrebbe pure:

si ha infine:

$$
\mathrm{R}=\frac{1}{p} n^{\prime} \mathrm{M}\left(1+\frac{\mathrm{G}_{2}}{\mathrm{~S}_{1}}\right) \underset{r_{1}}{\mathrm{R}_{1}^{\prime}}
$$

$$
\mathrm{R}=\mathrm{M} V n n^{\prime}\left(1+\gamma_{1}\right) \overline{\left(1+\gamma_{2}\right) \overline{\lambda_{1}}}
$$


dove

$$
\gamma_{1}=\frac{\mathrm{G}_{1}}{\mathrm{~S}}, \quad \gamma_{9}=\frac{\mathrm{G}_{3}}{\mathrm{~S}_{1}} ; \quad \lambda=\frac{\mathrm{R}^{\prime}}{r} ; \quad \lambda_{1}=\frac{\mathrm{R}_{1}^{\prime}}{r_{1}} .
$$

Notando ancora che quando il circuito induttore $\dot{\theta}$ interrotto la differenza di potenziale tra $x$ e $\beta$ non conserva lo stesso valore $\mathrm{V}, \mathrm{ma}$ ne acquista uno nuovo $\mathrm{V}_{1}$, indicando con $f$ la frazione del tempo unita scelta, durante la quale i circuiti induttore e compensatore sono simultaneamente attivi, con $b$ il rapporto $\bar{V}^{\prime}$, con e la f. e. m. costante che puó ritenersi che alimenti il circuito compensatore, si ha:

$$
e=[f+b(1--f)] \mathrm{V}=\frac{1}{\phi} \cdot \mathrm{V}
$$

* quindi :

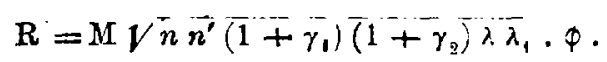

L'A. passa quindi a descrivere il metodo sperimentale tenuto per la determinazione delle quantità che compariscono in questa formula, $\theta$ ha trovato infine che alla temperatura di $17^{\circ}$ la cassetta da lui adoperata, valeva 1003,220 ohms internazionali, dando così per l'ohm un valore che, espresso in centimetri di mercurio, corrisponde a 106,20 centimetri.

\section{P. BACCEI}

Physikalische Zeitsch rift. N. ${ }^{i}$ 3-10, 1899.

Lonextz A. H. Contributo alla teoria del fenomeno di Zeeman (pp. 39-41).

RHCMBr.er I. Sulla formazione di figure spiraliformi nel bianco d' uova disseccato su un sostegno solido (pp. 41.43). - Durante il disseccamento il bianco d' uova si screpola suddividendosi in tanti poligoni, per lo più quadrilateri, in ognuno dei quali si forma una spirale più o meno regolare.

Puccraxtr Lutgr. Sugli spettri $d$ 'assorbimento di alcuni composti del carbonio nell' ultra rosso (pp. 49-51). - Lo radiazioni di una lampada ad incandescenza, attraversato uno spettrometro e il liquido assorbente, vengono esaminate mediante un radiometro.

Trova che l'alcool metilico ha tre bande d'assorbimento corrispondenti ad altrettante del ioduro di metile; il tolualo tre massimi di assorbimento coincidenti con altrettanti del benzolo, ecc. I risultati concordano col concetto che l'assorbinento dipenda dai gruppi di atomi che si trovano nella rnolecola, 
Batrell A. e Steranin A. Sulla misur della velocità dei raggi catodici (pp. 51-52). - Vedi N. Cimento, vol. 10, pag. 324).

Mater M. Diffrazione e lunghezza d'onda dei raggi di Röntgen (pp. 57.59). - Il metodo applicato è quello dovato al Fomm. Un tubo d'ottone porta agli estremi due fenditure parallele, regolabili, davauti alla prima sta la sorgente dei raggi, dopo la seconda una lastra sensibile, sulla quale si devono formare le frange. Ottiene per lunghezza d'onda $\mu, \mu 15$.

KAUPMAN W. Un modello meccanico per la rappresentazione del comportamento di un tubo Geissier (pp. 59-60). - E un modello idrodinamico molto semplice col quale si imitano alcuni noti fenomeni che avvengono nei tubi a vuoto, come:

1. Ja necessita di un certo potenziale per iniziare la corrente;

2." L'abbassanento che puó subire il potenziale una volta che è iniziata la scarica; ed altri analoghi.

Quin'T N. Determinazioni delle isoterme di mescolanze di acido cloridrico e di etino (pp. 65-66). - Per esaminare la teoria di Van der Waals relativa alla mescolanza di due sostanze, l'autore studia un miscuglio di etano (cemp. crit. $31^{\circ}, 88$ ) e di acido cloridrico, (temp. crit. $51^{\circ}, 3$ ), e ottioue una temperatura critica di $27^{\circ}, 2$, esterma a quella dei componenti.

Tofplik Max. Comportamento di una forma speciale della scarica elettrica nei ccımpi magnetici (pp. 66-67). - Comparirà piu in disteso nei Wied. Ann.

Dierericr C. Contributo "bllu teoria dells stato critico (pp. 7374). - La densita di molte sostanze allo stato critico è all'incirca 3,75 volto maggiore di quella ideale corrispondente alla legge del Boyle.

Invece se nella formula della teoria di Tan der Waals

$$
(p+\pi)(v-b)=\mathrm{R} \vartheta
$$

dove $\pi$ è la pressione di coesione $\theta b$ la correzione di volume, si suppone $b$ costante e $\pi=\frac{a}{v^{z}}$ dove $a$ è costante, si ricava come rapporto dei valori delle due densità 2,67 .

L'A. dimostra che non basta ammettere $b$ variabile, e propone due nodi per eliminare la contradizione.

Il primo consiste nell' ammettere $\pi=\frac{a t}{v^{\frac{5}{3}}}$; allora per $b=$ cost. il detto rapporto è 3,75 .

Nel secondo si fa l'ipotesi che la forza di coesione esistente tra le tnolecole, rallenti il movimento delle molecole che si muo- 
vono verso le pareti, attirandole verso l'interno, e che il lavoro contro detta forza sia proporrionale alla densita; nell' ipotesi di $b=$ cost. il rapporto noto ha il valore 3,695 .

Mooser J. Diamagnetism) dei mezzi dell'occhio (p. 75). L'A. trova che il cristallino di un occhio di bue è diamagnetico. La ricerca fu istituita allo scopo di applicare il magnetismo nella cura dei difetti della vista.

Schreber K. Il sistemx di misure dell' Ostwald e la dipendenza della tensione superficiale dal potenziale elettrico (pp. 75.76). - L'A. discute brevemente sui vantaggi che il sistema di misure dell' Ostwald ha sul sistema assoluto.

Quindi fa un'applicazione dei concetti svolti, cercando la relazione che deve sussistere tra potenziale eletrico e l'energia superficiale, allo scopo di determinare l' uaità di potenziale.

Con un metodo differenziale molto sensibile riesce a dimo. strare che la relazione nel caso di bolle di sapone $\dot{e}$

$$
\alpha=x_{0}\left(1-c P^{2}\right)
$$

dove $x$ è la tensione superficiale e $\mathrm{P}$ il potenziale. In questa esprimendo $x$ in erg. per crn., $P$ in volta, si ha

$$
c=35.10^{i}
$$

Des Cotidres Th. Metodo per rendere indipendenti dagli spostamenti di fase tra corrente e ten vione le indicazioni dei wattometri elettrodinamici (pp. 76-77).

WULFF L. Sulle curve di recaione dell' Ostwald nel cromo (p. 81). - L'A. nota che nelle curve di renzione del cromo registrate col metodo dell' Ostwald, il ramo che sale lentamente è composto di varie onde secondarie facilmente riconoseibili.

ElcuhorN W. Resistenza del bismuto in campi magnelici variabili (pp. 81-83). - L'A. vuol determiuaro so la resistenza elettrica del bismuto posto in an campo magnetico assume istan. taneamente il valore cbe le spetta.

Perciò misura la resistenza di ana spirale di bismuto che fa rotare nel campo magnetico, e trova che dipende dalla velocità di rotazione, mostrando una specie di isteresi.

Klemencic Iqnaz. Sullo sviluppo di calore mediante le correnti di Foucault prodotte da oscillazioni molto rapide (pp. 83-84). - Un conduttore che si trovi entro un solonoide posto nel circuito di scarica di una bottiglia di Leyda, è percorso dalle correnti di Foucault. Mediante ternometri a nercurio con bulbo sferico l'A. trova che l'incremento della temperatura aumenta col decrescere del diametro. Costruendo poi dei termometri ad 
alcool contenenti fili di ferro trova che lo svilnppo di calore a parità di volume è maggiore nei fili più sottili.

KLEMexcic IGNaZ. Ricerche su magneti permanenti.

I. Sulla dipendenza del coefficiente di temperatura dal rap. porto delle dimensioni (pp. 89-90). - Esamina 18 magneti permanenti e rettilinei che distingue in quattro gruppi. Ne misura la conducibilita elettrica e i coefficienti di temperatura.

'Trova che il prodotto del coefficiente di temperatura per il rapporto delle dimensioni è press'a poco costante per ogni gruppo o le variazioni procedono in buona parte in senso contrario alle variazioni della resistenza elettrica specifica dei magneti.

II. Sull, dipendenza doi coefficienti $d^{\prime}$ induzione dal rapporto delle dimensioni (pp. 111-112). - Esamina i coefficienti d'induzione delle 18 sbarrette precedenti prima di maguetizzarle e dopo, per tre intensità differenti del campo.

I coefficienti d' induzione sono maggiori nello stato non magrnetico quando il rapporto tra le dimensioni è maggiore di 15 , e la differenza cresce con questo rapporto.

Ricerche apposite dimostrarono poi che il momento permanente di un magnete $\dot{e}$ maggiore quando si fa diminuire il campo lentamente, che quando lo si interrompe bruscamente. L'aumento è piccolissimo nei magneti ordinari, ma notevole nei magneti corti e grossi.

Meyer Stefan und R. v. Schweidler Egon. Sul comportamento del radio e del polonio nei campi magnetici.

I (pp. 90-91). - Gli A. studiano l'influenza di an campo maguetico sull'azione scaricatrice del cloruro di radio e bario sui corpi eleltirizzati, collocando nel campo magnetico una volta il conduttore unito all' elettroscopio, l'altra il preparato attivo.

Trovano nei due casi che il tempo di scarica aumenta enormemente.

L'azione del campo magnetico é molto minore sul carbonato di bario e radio, nulla sul nitrato di polonio e bismuto.

II. (pp. 113-114). - Posto il cloruro di bario e radio in un campo magnetico ed esamiuando le radiazioni con uno schermo trovano che i raggi paralleli"alle linee di forza non sono deviati, mentre gli altri sono costretti a rotare jntorno ad esse.

HaGA H. e WINo C. H. Diffrazione e lunghezza d' onda dei raggi di Röntgen (pp. 91-92). - Osservano che le frangie ottenute dal Maier (Vedi sopra) non sono reali ma provengono da una illusione ottica, come venne dimostrato dal Wind (vedi l'articolo di C. H. Wind). 
Sommaremd A. Sulla teoria della diffrazione dei raggi di Röntgen (pp. 105-111).

WiND C. H. Per la dimostrazione di una illusione ottica sco. perta da E. Mach (pp. 112-113). - Si nettano due fenditure parallele, alla distanza di un tro cm; sulla prima si faccia cadero l' imagine di una sorgente laminosa, che attraverso ad essa illumini la seconda, e ne dia un' imagine su uno schermo posto a 3 o 4 metri.

Se la larghezza della seconda fenditura è di 2 o $3 \mathrm{~mm}$. e si fa variare gradatanente la prima a partire da zero, si vedono dapprima le frauge di diffrazione, ben presto scompaiono queste e compaiono all'orlo dell' imagine due striscie chiare che si avvicinano alla parte contrale, ivi si sovrappongono, e dopo prosaguono il loro cammino.

Quoste striscie sono apparenti eccetto che quando sono sovaapposte, a forse quando sono agli orli; infatti basta coprire con ano schermo annerito, anche notevolmente più basso dell'imagine della fenditura, parte di una delle due striscie più chiare perchè scompaiano entrainbe.

Lo stesso si ottiene sostituendo alla seconda fenditura un ago da calze.

Heydwfider Adock. Sulla pressione interna nelle soluzioni acquose (pp. 114-116).

A. A.MERIO.

\section{Wiedemann's Aunalen. Vol. LXV,}

N. 6. (continnazione) e N. 7. 1898.

HrTTORF W. Sulle proprietà elettromotrici del cromo (pp. 320343). - Da qualche tempo si prepara del cromo in massa intieramente fasa, grande quanto si vuole ed esente da carbone; il procedimento è per riduzione, per. nezzo dell'alluminio. Il motallo così ottenuto è grigio chiaro, fragile, possiede una viva lucentezza metallica, ed $\dot{e}$ buon conduttore dell'elettricità; alla rottura mostra cristalli assai voluminosi.

I'A. ha studiato un campione di questo corpo, dal punto di vista elettrico. Si sa che il cromo forma tre generi di composti ai quali corrispondono i tre ossidi $\mathrm{CrO}_{2} \mathrm{Cr}_{2} \mathrm{O}_{3}, \mathrm{CrO}_{3}$. Ora, quando si impiega il cromo come anodo, possono, secondo Ia temperatura e la natura del solvente, formarsi, anche collo stesso elettrolito, composti di ciascuno dei tre generi indicati. La superficie del cromo è allora in istati differenti, che persistono per un certo tempo dopo che il metallo é tolto dall' elettrolito. In questi tre stati il cromo possiede proprietà cosi differenti, come se si trat- 
tasse di tre metalli distinti. L'A. indica minutamente queste pro. prieta.

Konnstamm Р'H. о Cohen Е. Siludi fisico-chimici sull'elemen!o normale di Weston (pp. 344-357). - Jeager o Wachsmuth hanno dato come espressione della forza elettromotrice dell' elemento campione al cadmio:

$$
\mathrm{E}_{2}=1,0190-3,8 \times 10^{-5}(t-20)-0,065 \times 10^{-5}(t-20)^{2} .
$$

Questa formula è applicabile fra $5^{0}$ e $25^{n}$, ma questi fisici avevano osservato che fir $0^{\circ}$ e $15^{\circ}$ alcuni elementi hanno una forza elettromotrice suporiore di circa $1 / 1000$ di volta rispetto a quella di altri che corrisponde alla formula precedente.

Gli A. hanno stabilito con vari procedinenti che queste differenze sono dovute all' esistenza d' una modificazione subita verso i $15^{0}$ dai sali di cadmio che tanno parte dell' elemento.

Bisognerà dunque in pratica impiegare questo campione sol. tanto al di sopra di $15^{\circ}$; in tal caso il campione al cadıio $\dot{\theta}$, sotto tutti i riguardi, preferibile al campione Clark.

Braun F. Nota sulla termofonia (pp. 30̃8-360).

Brata F. Sopra l' emissione della luce su alcuni elettrodi negli elettroliti (pp. 361-361). - Facendo passare una corrente alternata attraverso una soluzione di acido solforico per mezzo di un elettrodo di platino e di uno d'alluminio, si osserva, all'oscuro, che quest' ulcimo elettrodo emette uniformemente su tutta la sua superficie una lace fosforescente bianca o rossa giallastra. Quando la densità della corrente è più grande, la luce diviene bluastra e si notano sulla lastra alcuni punti intensamente luminosi.

Studiando il fenomeno con uno specchio girante si nota una serio di immagini brillanti, nettamente separate, che corrispondono alle fasi della corrente per le quali l'elettrodo d'alluminio è catodo. Si potrebbe utilizzare questo fatto per riconoscere le differenze di fase di più correnti.

L'A. ha osservato questo fenomeno anche col magnesio e collo zinco in soluzioni convenienti; ma nel caso dello zinco l'ap-. parizione della luce avviene quando esso d̀ anodo.

Braú F. Sopra un criterio per riconoscere se uno strato superficiale conduttore è aderente, e sopra la tensione di vapore di tali strati (pp. 365-368). - L'A. osservó già ohe la pellicola d'acqua che si forma, all' umiditá, sopra un cristallo di gesso, dà un residuo elettrico che tende verso il massimo di polarizzazione corrispondente, quando lo spessore del deposito cresce, pur restando piccolissimo. Ammise allora per interpetrare l'esistenza di questo 
residuo, l'ipotesi che questo strato superficiale costituisse una pellicola aderente.

Per controllare l'esattezza di quest' ipotesi l'A. studia ora nello stesso modo il salgemma che, essendo solubilissino, deve senza dubbio formare nell' arja umida una pellicola liquida veramente aderente. In tal caso il fenomeno residuo scompare quasi del tutto.

L'A. ne conclude che nel caso del gesso non si ha una pellicola veramente aderente.

Lo studio della tensione di vapore permette di risolvere la stessa questione.

BraUN F. I raggi catodici sono soggetti alla rotazione unipolare? (pp. 368-371). - Le esperienze fatte dall'A. non sembrano favorevoli all'esistenza della rotazione di un fascio catodico intorno al polo di una calamita. Secondo l'A., i raggi catodici si compor. terebbero in un campo magnetico come correnti elettriche fisse per una estremità al catodo, elastiche, e tendenti a prendere una lunghezza massima. La rotazione sarebbe impedita dall' esser fisse per una delle loro estremitá.

Bratin F. Risposta (pp. 372-373).

DUANs W. Sulle pile termoelettriche elettrolitiche (pp. 374402). - L'A. si è proposto di verificare sperimentalmente le formule di Nernst a Planck sulla teoria della pressione ostaotica e sul trasporto degli ioni, per mezzo delie quali si puó rappresentare la forza elettromotrice delle coppie termoelettriche costituite da due elettroliti.

A questo scopo, per avvicinarsi alle condizioni ideali supposte da queste teorie, si è servito di soluzioni diluitissime.

Quando si tratta di due soluzioni diversamente concentrate di una medesima sostanza, le forze elettromotrici termoelettriche osservate presentano un accordo socidisfacente colle formule.

Ha quando si tratta di due soluzioni egualmente concentrate, ma di due sostanze differenti, fra l'esperienza e il calcolo c'é disaccordo completo. Bisogna allora per conciliare i risultati osservati colla teoria supporre dulle forze elettromotrici differenti da quelle che agiscono alla superficie di separazione dei due elettroliti. Quaste forze supplementari si eserciterebbero sugli ioni, lungo $i$ conduttori elettrolitici secondo i quali hanno luogo cadute di potenziale.

Du BoIs H. Sui prelesi schermi tangenziali (pp. 403.407). L'A. fa un esame critico dei vari lavori relativi a questa quistione. Riassumendo si può dire che un campo magnetico non $\dot{\theta}$ modificato per l'introduzione di una massa magnetica che occupa 
esattamente la posizione di un tubo di forza del campo. Particolarmente gli strati superficiali di nu cilindro indefinito (o d' un toro) magnetizzato parallelanente alle generatrici non esercitano sopra gli strati interni alcuna azione protettrice.

LATRILLE M. Sul pissaggio delle onde elettriche attraverso una fenditura (pp. 408-430). - L' apparecchio adoperato dall'A. si compone di an oscillatore a sfere, di un ricevitore costituito da un ooherer inserito nel circuito di una pila e di un galvano. metro, e di una fenditura che si pone fía l'oscillatore e il ricevitore e della quale si puó far variare a piacere l'orientazione, la lunghezza o la larghezza. Il coherer quantunque non sia uno strumento di misura quantitativa molto preciso pure, adoperato con alcune precanzioni può servire anche per uno studio quantitativo per mezzo delle deviazioni del galvanometro.

La fenditura si comporta come un reticolo di fili, cioè lascia passare quasi esclusivamente quella componente dell' oscillazione elettrica che d̀ normale alla sna lunghezza. Per una posizione de. terminata, se si aumenta la lunghezza della fenditura l'energia trasmessa auineuta dapprima con rapidità, poi sempre piủ lentameute. Se si aumenta iuvece la larghezza, l'energia trasmessa cresce dapprima lentamente, poi un po' più presto fino ad un certo limite.

BalfFManN W. La deviazione magnetica dei raggi catodici sottoposti all' influenza di un campo elettrostcatico (pp. 431-439). I raggi emessi da un catodo piano si fanno passare, nella direzione dell' asse, attraverso un cilindro che è caricato ad un potenziale di $\pm 3,2 \tilde{0} 0$ volt ed è circondato da un altro in comunicazione col suolo; quest' ultimo è alla sua volta circondato da un rocchetto attraversato dalla corrente. Poiché il potenziale nell' interno del primo cilindro è costante, la deviazjone elettromagnetica prodotta dal rocchetto si puó calcolare subito nella tèoria dell' emissione, che riceve una nuova conferma.

Se ne deduce per il rapporto fra la caricu elettrica portata da una particella e la massa di questa particella il valore $2,86 \times 10^{i}$ in unità (C. G. S).

WIEN W. Ricerche sulle scariche elettriche nei gas rarefatti (pp. 440-452). L'A. studia dapprima i raggi catodici col metodo di Lenard, in uno spazio vuoto separato dalla sorgente. Dalla loro deviazione per effetto di un cainpo elettrostatico noto l'A. ricava che la velocità è $\%$ di quella della luce e cbe il rapporto fra la massa materiale $\theta$ la carica elettrica di ciascuna particella è $5 \times 10^{-8}$.

L'A. studia in seguito i raggi canali di Goldstein, $\theta$ dalle sue osservazioni deduce analogamente che la velocità dei raggi 
$\dot{\theta}$ di $3,6 \times 10^{7} \mathrm{~cm}$. per secondo; $\theta$ che il rapporto fra la massa e la carica dielettrica delle particelle è $3,2 \times 10^{-3}$.

Fa quindi vedere come esistano dei raggi anodici e doi raggi canali anodici, di cui le proprietà sono analoghe ai raggi catodici. Terunina col mostrare l' analogia che passa fra i fenomeni che avvengono nei tubi di scarioa e quelli che arvengono in una vaschetta elettrolitica.

Graktz I. Ricerche sulla polarizzazione dei raggi di Röntgen (pp. 4553457). - Secondo Sobnke e Sohmidt, la luce emessa da alcuni cristalli biassici per fluorescenza o fosforescenza è polarizzata; l'A. ha cercato se $\mathrm{i}$ raggi $X$ emessi da un cristallo su cui cadeva un buon fascio di raggi catodici fossero polarizati. Fece passare questi raggi $X$ attraverso tormaline ma non poté con sicurezza costatare alcuna polarizzazione.

Pocknls F. Determinazione dell' intensitì massima delle cor. renti di scarica per mezzo della loro azione magnetizzante (pp. 458. 475). - Si amınette che il coefficiente di suscettibilità del ferro in un campo che varia rapidamente, come sarebbe il campo d'un rocchetto atcraversato da una scarica, sia lo stesso come se il cainpo magnetizzante variasse lentamente.

Per evitare l'azione di correnti indirette, si prende come corpo magnetico il basalto che contiene dei grani di ferro disso. minati in una massa isolante e per il quale la forza coercitiva $\theta$ la magnetizzazione rimaneate sono considerevoli; dal momento residuo e dall' ipotesi precedente si può dedurre sperimentalmente $l$ 'intensità massima.

D'altia parte si puó calcolare questa intensità servendosi delle formule di W. Thomson per una scarica data; ordinariamente si adopera la scarica continua.

Si trova questo resultato curioso che l'intensità calcolata è, in generale, inferiore a quella che è data dall'osservazione.

MüLleR E. Una nuova pompa a mercurio di Sprengel (pp. 476-478).

Havswam H. Sopra un perfezionamento all' interruttore a mercurio di Hofmeister (pp. 479-480).

DrtDe P. Sulla misura delle lunghezze dell' onde elettriche per mezzo del tubo a interferenza di Quinke (pp. 481-498). - Le onde elettriche inviate da un eccitatore Righi penetrano in parto in un tubo metallico che si biforca in due rami i quali terminano con una stessa tubulatura $t$; uno dei rami ha una langhezan costaute, l'altro una lunghezza variabile, come il tubo di König per misurare la lunghezza dello onde acustiche: se i cammini percorsi dalle due porzioni di onda differiscono di un numero 
pari o dispari di mezze lunghezze d'onda, queste due porzioni d'onda si sommeranno o si contrasteranno, e all' estremità $t$ si osserverá un massimo o un minimo dell' aziono elettrica.

Per svelare quest' azione l'A. adopera un coherer analogo a quello di Marconi.

Il risultato più noterole è che le lunghezze d'onda delle onde inviate da un eccitatore Righi e misurate col tubo di Quinke, dipendono dal diametro di questo.

Nelle ricerche fatte dall'A. la lunghezza d'onda è sansibilmunte il doppio del diametro del tubo.

DRUDe P. Sopra l'assorbimento di corte onde elettriche per parte dell acqux (pp. 499-503). - Le onde elettriche ottenute con un oscillatore Righi si fanno passare atliraverso una vasca di vetro piena d'acqua e le cui faccie sono formate da lastre di vetro ben parallole. Par nezzo di un coherer munito del suo galvanometro si misura l'energia delle onde attraversate.

L' energia, secondo le esperienze dell' A., decresce in modo continuo colla lunghezza attraversata, ciò che non si può spiegare che con un assorbimento.

L'A. conclude che l'assorbimento elettrico dell' acqua cresce quando la lunghezza d'onda diminuisce; esso è notevolissimo per $\lambda=10 \mathrm{~cm}$.

LeNaRD P. Sul comportamento dei raggi catodici paralleli alla forzx elettric $i$ (pp. 504-510). - L'A. fa passare i raggi catodici attraverso un condensatore posto nella direzione delle linee di forza e misura la deviazione di essi sotto l'azione di un campo magnetico e di un campo elettrico:

L'A. trova che questa deviazione è più grande o più piccola secondo che il raggio e il campo elettrico nel condensatore sono diretti nello stesso senso 0 in senso inverso.

Wennelt A. Sullo spazio catodico oscuro (pp. 511-542). Queste ricercho dimostrano che lo spazio catodico oscuro si comporta rispetto alla scarica come un dielettrico, per es. paraffina; se l' anodo e il catodo sono separati completaneute dallo spazio catodico oscuro, la scarica è nettamente disruttiva; in particolare, essa presenta le proprietà della scintilla in certe esperienze hertziane.

Già Ebert e Wiedemann avevano mostrato che se si sostituiva alla scintilla dell' eccitatore un tubo di Geissler, il sistema di fili di Lecher perdeva ogai traccia di risuonanza, qualunque fosso il potenziale esplosivo; ora l' $\mathrm{A}$. dimostra che, diminuendo sufficientemente la pressione nel tubo, la risuonanza ricompare con intensità. L'A. fa altre esperienze per confermare la sua proposizione. 
In un' altra serie di esperienze egli sposta il catodo o l'anodo, uno rispetto all' altro, e misura il potenziale esplosivo. $L$ ' anodo $\dot{e}$ un punto e il catodo un piano, o reciprocamente. Si trova fra gli altri, questo resultato curioso, che una diminuzione della distanza esplosiva aumenta il potenzialo esplosivo, quando l' anodo è nello spazio oscuro.

SWYNGEDatw R. Sulla scarica per scintille. Risposta a G. Jiaumann (pp. 543-552).

WESENDONCK K. Sopra l' azione dei gas delle fiamme sulle scariche elettriche luminose (pp. 553-566). - $\dot{\mathrm{E}}$ stato osservato cho gli alti camini raramente sono colpiti dal fulmine; ora l'A. ha cercato se questa particolarit fosse dovuta ad un' azione specialo dei gas provenienti dalla combustione. Egli si servi di un sistema di apparecchi, rappresentanti una nube carica in presenza di un camino munito di parafulmine. Dalle sue osservazioni risulta che invece di avere una influenza preservatrice, i gas che provengono dalle combustioni favoriscono la trasformazione delle scariche elettriche silenziose in scariche per scintille. La questione però è assai complessa, perchè gli effetti osservati variano colla temperatura e con la velocità con oui escono dalla sommità del camino i gas della combustione.

Arons L. Osservazione microscopica dei contatti variubili (coherer) (pp. 567-571). - L'A. si è servito del microsoopio per decidere se la variazione di resistenza delle polveri metalliche sotto l'influenza delle oscillazioni elottriche proviene da un seunplice fenomeno meccanico di contatto o da un fenomeno elettrico della natura della scintilla. Il suo coherer consisteva in un foglio di stagnola avente un picolo taglio sul quale venne posta una piccolissima quantità di polvere metallica; questa polvere era coperta con una goccia di balsamo del Canadà $\theta$ il tutto era posto sotto una lastrina di vetro. Sottomettendo questo coherer all' azione delle onde elattriche prodotte in un sistema di fili di Lecher si osservano al microscopio dei movimenti violenti delle particelle metalliche o dello vive scintille.

JaEgkR W. a Lindeck ST. Sulla costanza delle resistenze normali in manganina (pp. 572-599). - L'A. costrai cinque cam. pioni prototipi di resistenze in morcurio, oltre un gran numero di copie in mercurio e un più gran aumero di copie in filo di manganina.

La manganina presenta, coine è noto, un debolissimo coefficiente di temperatura. Durante ja anni la costanza delle copie di manganina si è unostrata superiore a quella delle copie di mer- 
curio; salvo poche eccezioni le resistenze di manganina uon hanno variato in tutto questo tempo che di alcuni centomillesini.

Arons 1. Soluzione analitica semplice di un caso schematico dell' effetto degli schermi elettromagnetici (pp. 590-594).

Trowbridge A. Sulla dispersione della silvina e il potere ri. flettore dei metalli (pp. 595̃.620).

Wiтrsa A. Sulla galvanometria delle scariche rapide, sotto forma di urti (pp. 621-628). - L'A. applisa il galvanometro di Wiedemann alla misura delle correnti di scarica rapidissime alle quali corrispondono le scintille delle macohine ad influenza a dischi multipli. Egli utilizza a questo scopo i metodi detti di moltiplicazione $\theta$ di riflessione che consistono nel produrre l' inversione automatica delle correnti nel moltiplicatore del galvanonetro a fasi determinato dell' oscillazioni dell' ago.

Questi procedimenti hanno dato all' $A$. buoni risultati; non solamente le elongaxioni si osservano con più facilità, ma quando le correnti che si studiano sono più o meno variabili si ottengono cosi delle buone medie.

MaRgUles M. Soluzione del platino e dell' oro negli elettroliti (pp. 629.634).

Sohadfelberger W. Osservazioni sul lavoro di Quinke: La viscosità dei liquidi isolanti in in campo elettrics costante (pp. 635-640). - Si ammette in generale che i dielettrici sottoposti a una polarizzazione alternativa manifestino fenomeni d'isteresi. Secondo l'A., l'ammortizzamento delle oscillazioni di una sfera isolante in un campo elettrico costante sarebbe dovuto, se non totalmente, almeno in gran parte, all' istoresi dielettrica del mezzo. Segue una spiegazione dettagliata.

Stumpr C. a Meyer M. Replica (pp. 641-644).

Meldw E. Replica al lavoro di Appun: Sulla determinazione delle altezze dei suoni acutissimi (pp. 645-647).

Schreber K. Sulla temperatura assoluta (pp 648-654).

Staigmülı ER H. Contributo alla teoria cinetica dei gas poliatomici (pp. 655-669).

StaigmültuR H. Sopra un metolo teorico che dà il valore della costante dellc legge di Dulong e Petit (pp. 670-672). - L'A. mostra come la teoria cinetica dei gas conduce a una espressione della legge di Dulong e Petit sui calori specifici.

M. Pandolfi. 